\title{
Trockenpulverinhalation bei Mukoviszidose
}

\author{
Dry Powder Inhalers in Cystic Fibrosis
}

eingereicht 21.12.2013 akzeptiert nach Revision 18.2.2014

\section{Bibliografie}

DOI http://dx.doi.org/

10.1055/s-0034-1365321

Online-Publikation: 24.3.2014

Pneumologie 2014; 68: 378-385

(c) Georg Thieme Verlag KG

Stuttgart · New York

ISSN 0934-8387

Korrespondenzadresse

Prof. Dr. med.

Gratiana Steinkamp

Medizinisch-wissenschaftliches

Publizieren

Reutzstraße 1

19055 Schwerin

steinkamp@med-wiss.com

www.med-wiss.com

\section{Zusammenfassung \\ $\nabla$}

Die inhalative Anwendung von Medikamenten hat für die Dauertherapie bei Mukoviszidose von jeher einen großen Stellenwert. Dabei war die Feuchtinhalation die klassische Applikationsform. Das zähe Bronchialsekret soll durch gelöste lungengängige Partikel gelockert werden, sodass die Atemwege mit Husten oder mit Physiotherapie leichter gereinigt werden können. Die erforderlichen hohen Dosierungen der pseudomonaswirksamen inhalativen Antibiotika konnten früher nur mittels Feuchtverneblung von Lösungen erreicht werden. Seit wenigen Jahren sind inhalative Präparate in Pulverform speziell für Mukoviszidose verfügbar. Die neu entwickelten Arzneimittel und Inhalatoren unterscheiden sich deutlich von den herkömmlichen Pulverpräparaten zur Therapie chronisch obstruktiver Atemwegserkrankungen. Zwei inhalative Antibiotika in Pulverform, Tobramycin und Colistin, sowie das osmotisch wirksame und schleimlösende Mannitol werden in diesem Artikel vorgestellt. Präsentiert werden technologische Aspekte, Studiendaten zu Wirksamkeit und Verträglichkeit sowie die praktische Anwendung dieser neuen Arzneimittel.

\section{Einleitung}

$\nabla$

Die Vernebelung von Medikamenten gehört bei Mukoviszidose zum Therapiestandard. Die Befeuchtung der intrathorakalen Atemwege durch respirable Partikel von Medikamentenlösungen macht es leichter, den zähen Bronchialschleim zu lockern. Flüssigeres Sekret lässt sich leichter abhusten bzw. mit geeigneten Techniken der Atemphysiotherapie herausbringen. Auch die erforderlichen hohen Dosierungen bei den zur Behandlung der chronischen Lungeninfektion eingesetzten Antibiotika konnten früher nur mittels Feuchtverneblung von Lösungen erreicht werden. Arzneimittel zur Feuchtinhalation sind beispiels-

\section{Abstract \\ $\nabla$}

Inhaled medications play an important role in the daily treatment of patients with cystic fibrosis (CF). The classic route of administration was nebulisation via jet nebulisers. Respiratory delivery of fluid particles should loosen the viscid respiratory secretions, making airway clearance via cough or physiotherapy more efficient. Until recently, only jet nebulisers allowed to administer high doses of aerosolised antipseudomonal antibiotics. Powder inhalers for the treatment of cystic fibrosis have recently been made available. The newly developed powders and inhalers differ considerably from conventional dry powder inhalers used for the treatment of chronic obstructive airway disease. The present article will review two inhaled antibiotics, i.e. tobramycin and colistin, and the hyperosmotic agent mannitol, which increases the hydration of the airways. Topics are particle engineering, efficacy and tolerability results from clinical trials, as well as functional and practical aspects related to these new drugs.

weise hypertone Kochsalzlösung, Dornase alfa, Bronchodilatatoren oder inhalative Antibiotika mit den Wirkstoffen Tobramycin, Colistin oder Aztreonam.

Seit wenigen Jahren sind inhalative Präparate in Pulverform speziell für Mukoviszidose verfügbar. Sie beseitigen Nachteile der Feuchtinhalation, wie geringe pulmonale Deposition, umständliche Handhabung und aufwändige Reinigung. Die Technologien zur Herstellung der neuen Präparate unterscheiden sich stark von denen herkömmlicher Pulverinhalatoren zur Therapie chronisch obstruktiver Atemwegserkrankungen. 


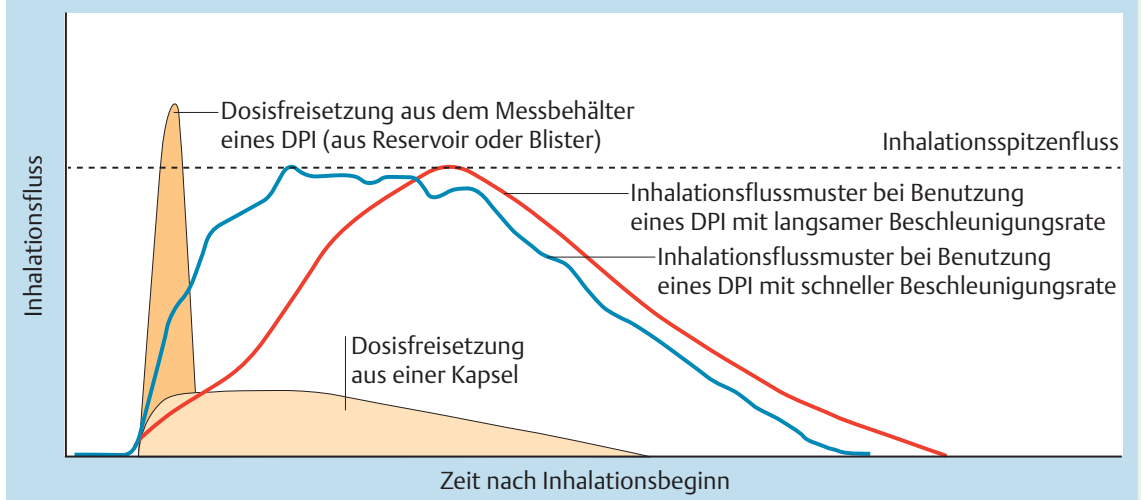

Abb. 1 Inspiratorische Flussmuster bei Trockenpulverinhalation [1].

In der folgenden Übersicht werden drei neue Präparate und ihre Besonderheiten vorgestellt. Neben Ergebnissen zur Wirksamkeit und Verträglichkeit der Arzneimittel geht es auch um praktische Aspekte der Anwendung.

\section{Trockenpulverinhalatoren}

Trockenpulverinhalatoren (dry powder inhaler, DPI) haben seit Jahrzehnten einen festen Platz in der Therapie obstruktiver Atemwegserkrankungen. Man unterscheidet drei Typen, je nachdem, wie das Medikament im Inhalator (Device) verwendet wird [1]. Reservoirbasierte Pulverinhalatoren wie der Turbohaler ${ }^{\circledR}$ enthalten einen größeren Medikamentenvorrat, der für einige Wochen Therapie ausreicht. Bei jeder Inhalation wird daraus mithilfe des Atemstroms eine Einzeldosis abgeschieden. Kapselbasierte Inhalatoren setzen Einzeldosen von Medikamenten frei. Diese werden entweder als einzelne Kapsel in den Inhalator eingefüllt (zum Beispiel Aerolizer ${ }^{\circledR}$ ), oder sie befinden sich in einem Blisterstreifen, aus dem die Einzeldosen nacheinander von Anwendung zu Anwendung freigegeben werden (zum Beispiel Diskus ${ }^{\circledR}$ ).

\section{Voraussetzungen und Einflussfaktoren}

Für die Anwendung von Pulverinhalatoren gelten bestimmte physikalische und physiologische Voraussetzungen. Der innere Widerstand des Gerätes hat Einfluss darauf, welchen inspiratorischen Fluss der Patient aufwenden muss, um genügend Partikel zu inhalieren. Bei Devices mit höherem Widerstand wie dem Turbohaler oder dem Diskus sind dazu inspiratorische Spitzenflüsse von etwa 601/min optimal. Junge Kinder oder Patienten mit stark eingeschränkter Lungenfunktion sind jedoch häufig nicht in der Lage, diese Flüsse zu generieren [2].

Pulverinhalatoren unterscheiden sich auch danach, welche Atemmanöver erforderlich sind, um respirable Partikel zu generieren. Beim Inhalieren kann eine schnelle oder eine langsame Beschleunigungsrate erforderlich sein [1]. Wenn die Dosis aus einem Reservoir des DPI freigesetzt werden muss, sind sofort nach Beginn der Inhalation hohe Inhalationsflüsse erforderlich (schnelle Beschleunigungsrate). Der Patient muss kräftig und schnell einatmen. Atmet er zu schwach ein, steigt der Inhalationsfluss zu langsam an, und es werden größere Mengen des Medikaments im Mund-Rachenraum anstatt in den Bronchien deponiert [3]. Bei Dosisfreisetzung aus einer Kapsel kann die Beschleunigungsrate dagegen geringer sein, und die Dosis wird über eine längere Zeit eingeatmet ( $\bullet$ Abb. 1).

\section{Feuchtinhalation von Antibiotika bei CF}

Bei Patienten mit Mukoviszidose und chronischer Pseudomonasinfektion der Lunge hat sich die Langzeitinhalation mit Antibiotika seit vielen Jahren etabliert [4]. Die ersten speziell für die inhalative Anwendung zugelassenen Antibiotika wurden mithilfe von Düsenverneblern inhaliert. Dies dauert pro Anwendung etwa 15 bis 20 Minuten. Hinzu kommt der zeitliche Aufwand für die Reinigung und Wartung des Gerätes. Bei der Inhalation über einen Kompressionsvernebler werden die Atemwege durch die Inhalationslösung befeuchtet, und es kann bereits ein Teil der Atemphysiotherapie durchgeführt werden.

Die neueren Membranvernebler haben diverse Vorteile gegenüber den klassischen Kompressionsverneblern. „VibrierendesNetz-Geräte“ wie z.B. der PARI eFlow ${ }^{\circledR}$ sind kleiner, besser transportabel, leiser und vernebeln das Medikament in kürzerer Zeit als die konventionellen Kompressionsvernebler [5].

\section{Pulverinhalation als Alternative}

Als Alternative zur Vernebelung wurden speziell für Mukoviszidose Pulverinhalatoren zur inhalativen Applikation der Antibiotika Tobramycin und Colistin entwickelt. Seit 2012 ist außerdem Mannitol-Pulver als Schleimlöser zum Inhalieren verfügbar. Die Pulverinhalation hat diverse Vorteile gegenüber der Feuchtinhalation über Kompressions- oder Membranvernebler ( Tab.1). Vor allem ist die Belastung des Patienten durch die Therapie geringer.

Tab. 1 Vorteile der Pulver- und der Feuchtinhalation.

\begin{tabular}{lc} 
Vorteile der Pulverinhalation & Vorteile der Feuchtinhalation \\
\hline - geringe Belastung des Patienten & - Befeuchtung der Atemwege \\
durch die Therapie & beim Inhalieren \\
- kurze Inhalationszeit & - geringe Anforderungen an die \\
- leises Inhalieren & Koordinationsfähigkeit des \\
- weder Stromanschluss noch & Patienten \\
Batterien erforderlich & - geringe Anforderungen an die \\
- in der Hosentasche zu verstauen & Einatemtechnik \\
- überall anzuwenden & - geringer inspiratorischer \\
- einfach zu reinigen & Druck ausreichend \\
- wartungsfrei & - geringer inspiratorischer \\
- konsistente Dosierung & Spitzenfluss ausreichend \\
- effiziente pulmonale Deposition & - geringer Hustenreiz \\
- keine zugesetzten Treibgase & - Inhalation kann in die Physio- \\
- kein Verlust des Medikaments in & therapie integriert werden \\
die Umgebung & - auch für junge Kinder geeignet \\
- keine Kontamination der Um- & - auch bei stark eingeschränkter \\
gebung durch das Arzneimittel & Lungenfunktion anwendbar
\end{tabular}


Um eine gute antimikrobielle Wirkung zu erzielen, sind relativ hohe Antibiotikadosen erforderlich. Die klassische TobramycinInhalationslösung enthält pro Inhalation $300 \mathrm{mg}$ Tobramycin und die Colistin-Lösung (1 Mio. IU) 80 mg Colistimethat-Natrium. In Milligramm sind diese Wirkstoffmengen mehr als hundertmal höher als die bei der Therapie des Asthmas oder der COPD üblichen Einzeldosen (z. B. 0,1 mg bei inhalativen Steroiden oder 0,05 $\mathrm{mg}$ bei Bronchodilatatoren). Auch beim Mannitol-Pulver liegt die Einzeldosis von $40 \mathrm{mg}$ um Größenordnungen höher als bei herkömmlichen Pulverpräparaten.

Einschränkungen bei konventionellen Trockenpulvern Bei konventionellen Trockenpulvern ist die Größe der Einzeldosis begrenzt. Die klassische Herstellungsmethode beruht darauf, dass große Medikamentenkristalle solange vermahlen (mikronisiert) werden, bis lungengängige Partikel in einer Größe von 1 bis $5 \mu \mathrm{m}$ entstehen. Die beim Vermahlen resultierenden Medikamententeilchen sind unterschiedlich groß und haben als Kristalle kantige, glatte Oberflächen. Zwischen benachbarten Partikeln bestehen starke elektrostatische Kräfte, sodass die Partikel dazu neigen, zusammenzuklumpen. Um dies zu verhindern, werden dem Arzneimittel Trägerpartikel hinzugefügt, meist Laktose [3]. Das Verhältnis zwischen Trägerpartikeln und Medikament beträgt bei klassischen Pulvern etwa 65 zu 1 Gewichtsanteile. Um die Medikamentenpartikel von der Trägersubstanz zu separieren, muss der Patient kräftig einatmen und einen ausreichenden Einatemfluss aufbauen. Dennoch gelangt bei der Inhalation nicht der gesamte Wirkstoff in die Lunge, sondern nur 10-30\% des deklarierten Wertes. Damit liegt die maximale Lungendosis bei wenigen Milligramm pro Inhalation [6].

\section{Neue Technologien zur Herstellung respirabler Partikel („engineered particles“)}

Aufgrund technischer Gegebenheiten kommt nicht jeder Typ Inhaliergerät für jede zu applizierende Inhalationsdosis infrage. Weder herkömmliche Trockenpulverinhalatoren noch Dosieraerosole können Einzeldosen von mehr als $2 \mathrm{mg}$ abgeben [7]. Um höhere Dosen inhalierbar zu machen, wurden daher neue Pulvertechnologien entwickelt.

\section{Die PulmoSphere ${ }^{\mathrm{TM}}$ Technologie}

PulmoSpheres sehen unter dem Mikroskop wie hohle, durchlöcherte Kügelchen aus. Sie haben einen mittleren Massendurchmesser von 1,7 bis 2,7 $\mathrm{m}$, sind leicht und sehr gut lungengängig [1]. Mit dem Einatemstrom gelangen die PulmoSpheres tief in den Bronchialbaum hinein. Dazu reichen bereits geringere inspiratorische Flussraten aus. Anders als die kristallinen, mikronisierten Partikel neigen die porösen Bällchen nicht zum Aggregieren.

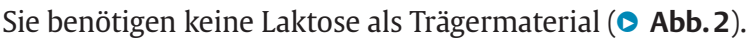

PulmoSpheres für Tobramycin werden mit einem Sprühtrocknungsverfahren hergestellt [6]. Dabei entstehen hohle Kügelchen mit zahlreichen Poren. Diese Partikel bestehen zu mehr als 90\% aus reinem Medikamentenwirkstoff ohne Trägermaterial. Der mediane geometrische Durchmesser beträgt 1,7-2,7 $\mu \mathrm{m}$ [6]. Aufgrund der leichten porösen Struktur ergeben sich gute aerodynamische Eigenschaften, die zu einer vergleichsweisen guten Deposition auch in der Lungenperipherie führen [8].

Das Präparat TOBI Podhaler ${ }^{\circledR}$ wurde im Juli 2011 in Deutschland und Europa für Mukoviszidose-Patienten ab 6 Jahren zugelassen [9]. Indikation ist die chronische Infektion mit Pseudomonas

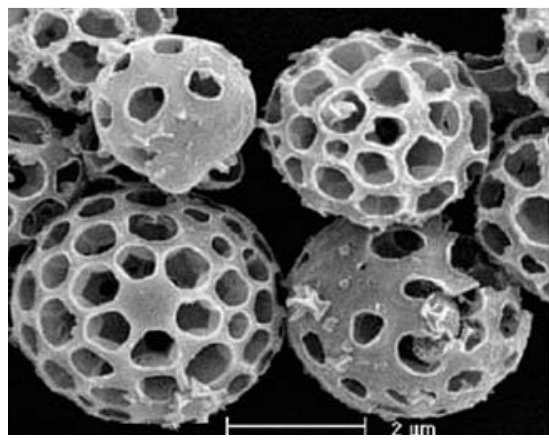

Abb.2 PulmoSpheres sind homogene Partikel, in die Wirkstoffe gepackt wurden. Sie können exzellent intrapulmonal deponiert werden. Mit freundlicher Genehmigung der Novartis Pharma GmbH.

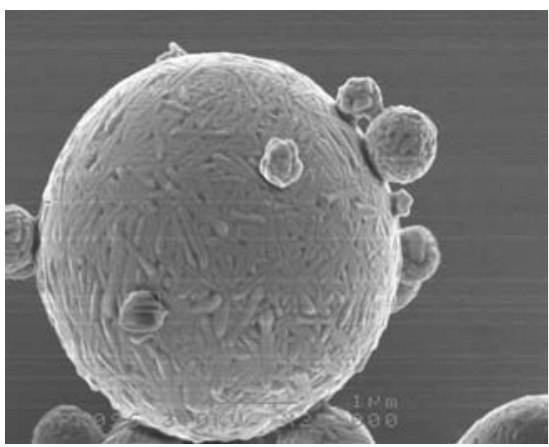

Abb.3 Sprühgetrocknetes Mannitol. Mit freundlicher Genehmigung von Pharmaxis Pharmaceuticals Ltd, UK.

aeruginosa. Die Einzeldosis von $112 \mathrm{mg}$, also 4 Kapseln à $28 \mathrm{mg}$, entspricht bei Mukoviszidosepatienten der $300 \mathrm{mg}$ Dosis der Inhalationslösung [10]. Der pulmonal deponierte Anteil von Tobramycin war bei Gesunden mit 34,2\% rund dreimal höher als bei der Inhalationslösung, und die Variabilität zwischen den Versuchspersonen betrug nur 17\% [8]. Die maximalen Sputumkonzentrationen von Tobramycin lagen mit rund $1000 \mu \mathrm{g}$ pro Gramm Sputum bis zu 100fach höher als die bei $P$. aeruginosa normalerweise zu beobachtenden minimalen Hemmkonzentrationen [8]. Ein kleiner Teil des Antibiotikums wird resorbiert, und nach $80 \mathrm{mg}$ Tobramycin-Pulver lagen die maximalen Serum-Konzentrationen bei durchschnittlich $0,60 \mu \mathrm{g} / \mathrm{ml}$ im Vergleich zu 0,28 $\mu \mathrm{g} / \mathrm{ml}$ nach $300 \mathrm{mg}$ Tobramycin-Inhalationslösung [8].

\section{Sprühgetrocknetes Mannitol}

Der natürlich vorkommende Zuckeralkohol Mannitol wurde von vornherein als Pulverinhalat entwickelt. Bei seiner Herstellung wird das kristalline Mannitol-Pulver mit einem Sprühtrocknungsverfahren in kugelförmige Partikel mit einem mittleren Durchmesser von etwa $3 \mu \mathrm{m}$ gebracht $(\bullet$ Abb.3). Das Arzneimittel enthält keine weiteren Bestandteile, sondern ausschließlich Mannitol. Bei Gesunden wurden zwischen 13 und 31\% der Mannitol-Dosis in der Lunge deponiert [12].

Bei den ersten Anwendungen in Australien verwendete man Mannitol zur bronchialen Provokationstestung bei Verdacht auf Asthma bronchiale [13]. Wie dieselbe Forschergruppe feststellte, fördert Mannitol Trockenpulver bei Gesunden und bei Asthmatikern die mukoziliäre Clearance [14]. Die erste Pilotstudie bei Mukoviszidose wurde 1999 publiziert [15]. Die mit einer Gammakamera gemessene Clearance stieg nach Inhalation von $300 \mathrm{mg}$ Mannitol signifikant stärker an als nach der Kontrollmedikation, und sie lag in einem ähnlichen Bereich wie nach Vernebelung von $6 \%$ iger Kochsalzlösung.

Da Mannitol nicht nur bei Asthma, sondern auch bei CF zur Bronchokonstriktion führen kann, ist vor Behandlungsbeginn ein „Initialdosis-Test“ zwingend erforderlich. Dabei werden aufsteigen- 


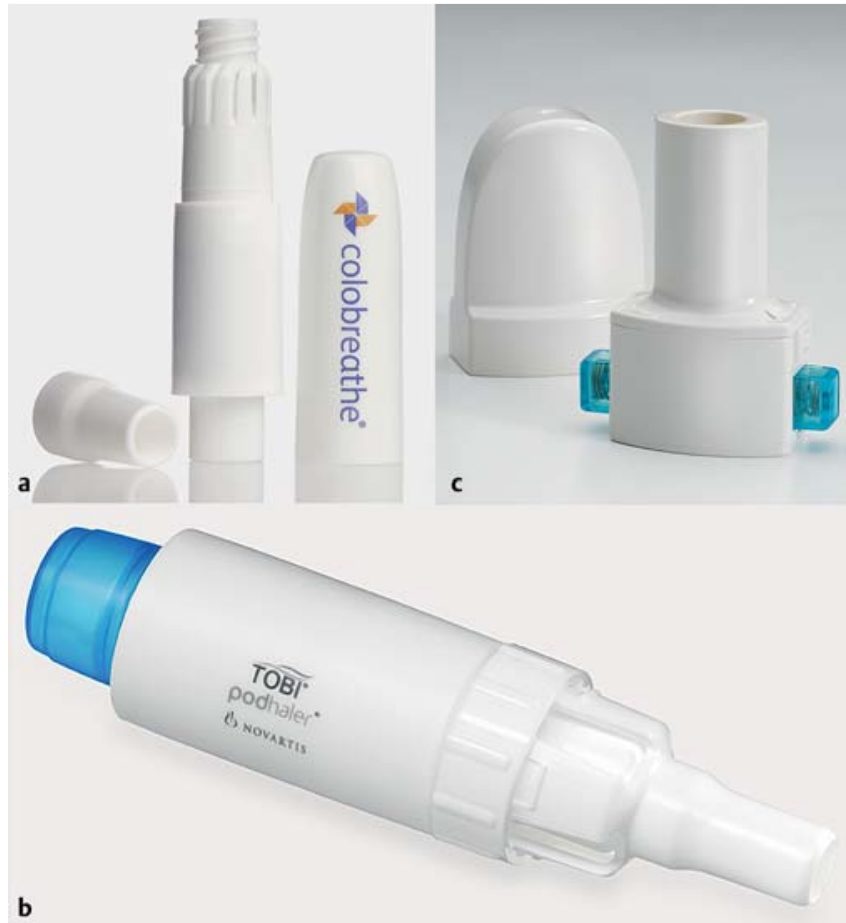

Abb.4 Pulverinhalatoren für Colistin (Colobreathe ${ }^{\circledR}$ ), Tobramycin (TOBI ${ }^{\circledR}$ Podhaler $^{\circledR}$ ) und Mannitol (Bronchito ${ }^{\circledR}$ )

a Colobreathe ${ }^{\circledR}$ Turbospin Inhaler. Mit freundlicher Genehmigung von Forest Laboratories, Berlin.

b TOB ${ }^{\circledR}$ Podhaler ${ }^{\circledR}$. Mit freundlicher Genehmigung von Novartis Pharma GmbH, Nürnberg

c Bronchitol ${ }^{\circledR}$ Inhaler. Mit freundlicher Genehmigung von Pharmaxis Pharmaceuticals Ltd, UK.

de Dosen von $40 \mathrm{mg}, 80 \mathrm{mg}, 120 \mathrm{mg}$ und $160 \mathrm{mg}$ Mannitol unter Überwachung von Spirometrie und Sauerstoffsättigung appliziert [16].

Das Präparat Bronchitol ${ }^{\circledR}$ wurde im April 2012 in Deutschland für Erwachsene ab 18 Jahren zugelassen [16]. Studien bei Kindern und Jugendlichen sind auf dem Weg. Bronchitol ${ }^{\circledR}$ soll zusätzlich zum besten Therapiestandard angewendet werden.

\section{Neue Pulverinhalatoren}

$\nabla$

Zusätzlich zu den neuen Technologien für die Herstellung der Arzneistoffe wurden auch neue Inhalationsgeräte entwickelt, mit denen größere Einzeldosen von Pulver inhaliert werden können als mit konventionellen Pulverinhalatoren ( $\bullet$ Abb.4).

Der T-326 Pulverinhalator wird als kapselbasierter Podhaler ${ }^{\circledR}$ zum Inhalieren von Tobramycin-Pulver (TOBI Podhaler ${ }^{\circledR}$ ) verwendet. Jede Medikamentenkapsel enthält $28 \mathrm{mg}$ Tobramycin. Pro Anwendung werden nacheinander vier Kapseln inhaliert, die jeweils in einem separaten Blisterkompartiment abgefüllt sind.

Die meisten Patienten können die Kapsel mit einer einzigen Inhalation entleeren. Wie andere Pulverinhalatoren funktioniert der T-326 rein mechanisch und benötigt keine externen Energiequellen. Er ist klein und leicht und passt in die Jackentasche. Die Reinigung ist einfach, denn nach der Inhalation muss lediglich das Mundstück mit einem trockenen Tuch abgewischt werden. Da jede Dosis in einer separaten Kapsel verpackt ist, ist das Medikament gut vor Feuchtigkeit geschützt.

Das Antibiotikum Ciprofloxacin wird derzeit ebenfalls für die Trockenpulverinhalation entwickelt. Auch hier wird der T-326 Inhalator und die PulmoSphere Technologie verwendet [17]. Zur Inhalation von Colistimethat-Natrium (Colobreathe ${ }^{\circledR}$ ), der Prodrug von Colistin, dient der Turbospin ${ }^{\circledR}$ Pulverinhalator. Er ist ein Vorläufer des T-326 Inhalators [18]. Die Einzeldosis pro Kapsel beträgt 1.662.500 IU Colistimethat-Natrium entsprechend $125 \mathrm{mg}$. Wegen dieser hohen Wirkstoffmenge reicht pro Anwendung eine Kapsel aus. Zu diesem Device wurden bisher kaum wissenschaftliche Daten veröffentlicht [18]. Hinsichtlich des inneren Widerstandes des Gerätes beträgt die Flussrate bei einem Druckabfall von $4 \mathrm{kPa}$ beim Turbospin 731/min [Forest Laboratories Europe, persönliche Kommunikation].

In den meisten klinischen Studien mit Mannitol wurde als Inhalator der Osmohaler (RS01, Plastiape, Italien) oder ein Prototyp verwendet. Das Präparat Bronchitol ${ }^{\circledR}$ wird über diesen Inhalator appliziert. Er gehört zur selben Serie wie der Aerolizer ${ }^{\circledR}[19]$ und sieht auch sehr ähnlich aus, die beiden Inhalatoren sind jedoch nicht identisch. Pro Anwendung werden nacheinander zehn Hartkapseln à $40 \mathrm{mg}$ Mannitol inhaliert. Studien bei gesunden Versuchspersonen zeigten, dass etwa 40\% der Dosis pulmonal deponiert wurden, wenn die Partikel einen Durchmesser von 2 oder $3 \mu \mathrm{m}$ hatten [20].

Angaben zur pulmonalen Deposition und zu maximalen Serumund Sputumkonzentrationen bei Inhalation dieser Präparate sind in Tab. 2 zusammengefasst.

\section{Wirksamkeit der Pulverinhalation bei Mukoviszidose $\nabla$}

Zu jeder der drei Substanzen Mannitol, Tobramycin und Colistin wurden klinische Prüfungen der Phase 3 mit mehreren Hundert Teilnehmern publiziert, deren Hauptergebnisse hier zusammenfassend vorgestellt werden.

\section{Verbesserung der Lungenfunktion}

Die Veränderung der Einsekundenkapazität FEV1 wird von den Zulassungsbehörden als Studienendpunkt bei Mukoviszidose akzeptiert [21] und diente bei den klinischen Phase-3-Prüfungen der Trockenpulver-Präparate als Parameter für die Wirksamkeit des Arzneimittels. Bei Personen mit Mukoviszidose fällt die FEV1 im Krankheitsverlauf allmählich ab. Pro Jahr sind es durch-

\begin{tabular}{|llll|}
\hline & $\begin{array}{l}\text { Colistimethat-Natrium } \\
\left(\text { Colobreathe }^{\circledR}\right)\end{array}$ & $\begin{array}{l}\text { Tobramycin Pulver } \\
\left(\text { TOBI Podhaler }^{\circledR}\right)\end{array}$ & $\begin{array}{l}\text { Mannitol } \\
\left(\text { Bronchitol }{ }^{\circledR}\right)\end{array}$ \\
\hline pulmonale Deposition & $11,6 \%$ & $34 \%$ & $24,7 \%$ \\
\hline maximale Sputumkonzentration & $18,5 \mu \mathrm{g} / \mathrm{ml}$ & $1048 \mu \mathrm{g} / \mathrm{g}$ & $\mathrm{keine} \mathrm{Angabe}$ \\
\hline maximale Serumkonzentration & $0,0524 \mu \mathrm{g} / \mathrm{ml}($ Erw.) & $1,02 \mu \mathrm{g} / \mathrm{ml}$ & $>10 \mu \mathrm{g} / \mathrm{ml}$ \\
\hline innerer Widerstand des Inhalators & gering & gering & hoch \\
\hline
\end{tabular}

Tab. 2 Sputum- und Serumkonzentrationen der Pulverpräparate.

Quellen: Colobreathe ${ }^{\circledR}:$ [29], Davies 2004, [24]; Tobi Podhaler ${ }^{\circledR}:$ [8], [9]; Bronchito ${ }^{\circledR}:$ [30], [16], [12], [20] 
Tab.3 Verträglichkeit der Pulverinhalation und FEV1-Verbesserung in den großen Phase-3-Studien.

\begin{tabular}{|c|c|c|c|c|c|c|c|c|c|c|}
\hline & \multicolumn{2}{|c|}{ Colistimethat-Natrium } & \multicolumn{6}{|c|}{ Tobramycin Pulver } & \multicolumn{2}{|l|}{ Mannitol } \\
\hline Studien-Acronym & \multicolumn{2}{|l|}{ FREEDOM } & \multicolumn{2}{|l|}{ EAGER } & \multicolumn{2}{|l|}{ EVOLVE } & \multicolumn{2}{|c|}{ EDIT } & \multicolumn{2}{|c|}{ CF 301 und CF 302} \\
\hline Erstautor & \multicolumn{2}{|c|}{ Schuster 2012} & \multicolumn{2}{|c|}{ Konstan 2011} & \multicolumn{2}{|c|}{ Konstan 2010} & \multicolumn{2}{|c|}{ Galeva 2013} & \multicolumn{2}{|c|}{ Bilton 2013} \\
\hline verblindet? & \multicolumn{2}{|l|}{ nein } & \multicolumn{2}{|l|}{ nein } & \multicolumn{2}{|c|}{ erste 4 Wochen ja } & \multicolumn{2}{|l|}{ ja } & \multicolumn{2}{|l|}{ ja } \\
\hline Dauer & \multicolumn{2}{|l|}{24 Wochen } & \multicolumn{2}{|c|}{24 Wochen } & \multicolumn{2}{|c|}{20 Wochen } & \multicolumn{2}{|c|}{28 Tage } & \multicolumn{2}{|c|}{26 Wochen } \\
\hline Gruppe & $\mathrm{CDPI}^{*}$ & TIS* & TIP* & TIS & TIP & Placebo & TIP & Placebo & Mannitol & Kontrolle \\
\hline Formulierung & Pulver & Lösung & Pulver & Lösung & Pulver & Pulver & Pulv & Pulver & Pulver & Pulver \\
\hline Einzeldosis & 1,6625 MU & $300 \mathrm{mg}$ & $112 \mathrm{mg}$ & $300 \mathrm{mg}$ & $112 \mathrm{mg}$ & & 112 & & $400 \mathrm{mg}$ & $50 \mathrm{mg}$ \\
\hline Zahl der Patienten (n) & 186 & 193 & 308 & 209 & 46 & 49 & 30 & 32 & 361 & 239 \\
\hline \multicolumn{11}{|l|}{ Wirksamkeit } \\
\hline $\begin{array}{l}\text { Delta FEV1 zwischen } \\
\text { den Studiengruppen } \\
\text { (relativ, \% des Solls) }\end{array}$ & \multicolumn{2}{|l|}{$-0,98$} & \multicolumn{2}{|l|}{1,1} & \multicolumn{2}{|c|}{13,3} & \multicolumn{2}{|c|}{5,9} & \multicolumn{2}{|l|}{3,6} \\
\hline \multicolumn{11}{|l|}{ Verträglichkeit } \\
\hline $\begin{array}{l}\text { unerwünschtes Ereignis } \\
\text { (UE, \% Patienten) }\end{array}$ & 94 & 89 & 90 & 84 & 50 & 76 & 27 & 34 & 88 & 90 \\
\hline $\begin{array}{l}\text { unerwünschte Arznei- } \\
\text { mittelwirkung (\%) }\end{array}$ & 82 & 47 & $\begin{array}{l}\text { Husten: } \\
25\end{array}$ & $\begin{array}{l}\text { Husten: } \\
4\end{array}$ & & & 17 & 6 & & \\
\hline $\begin{array}{l}\text { vorzeitiger Abbruch } \\
\text { wg. UE (\%) }\end{array}$ & 12 & 3 & 13 & 8 & 0 & 2 & 3 & 3 & 11 & 6 \\
\hline schwerwiegendes UE (\%) & 4 & 6 & 27 & 29 & 7 & 14 & 0 & 6 & 21 & 27 \\
\hline
\end{tabular}

* CDPI: Colistin Trockenpulver, TIP: Tobramycin Pulver, TIS: Tobramycin Lösung

schnittlich 1 bis $2 \%$ des Sollwerts, mit stärkeren Verlusten bei Patienten mit guter Lungenfunktion [22]. Daher sind nach medikamentöser Therapie selbst kleine Verbesserungen der FEV1 um wenige Prozent des Solls oder um absolut $70 \mathrm{ml}$ als Behandlungserfolg zu werten.

In den großen Mannitol-Studien wurden zweimal täglich $400 \mathrm{mg}$ über 26 Wochen im Vergleich zur Kontrollmedikation (je $50 \mathrm{mg}$ Mannitol) geprüft ( Tab.3). In der gepoolten Auswertung zweier Phase-3-Studien erzielten die Autoren für Mannitol $400 \mathrm{mg}$ eine Überlegenheit von $3,6 \%(p<0,001)$, bezogen auf die relative Veränderung der FEV1 [23]. Ausgedrückt in ml FEV1 betrug die Differenz zwischen den Gruppen 73,4 ml zugunsten des Verums $(\mathrm{p}<0,001)$.

In der Phase-3-Studie zu Colistimethat-Natrium Pulver erfolgte ein Vergleich zur Tobramycin-Lösung, die über einen Kompressionsvernebler inhaliert wurde [24]. Die Dosen betrugen $1.662 .500 \mathrm{IU}$ Colistimethat-Natrium bzw. $300 \mathrm{mg}$ Tobramycin zweimal täglich. Die klinische Prüfung verlief über 24 Wochen und war nicht verblindet. Alle Teilnehmer hatten vor Studienbeginn über mindestens zwei On-Off-Zyklen mit Tobramycin inhaliert. Colistin wurde täglich inhaliert, während bei TobramycinLösung das übliche 28 Tage On-Off-Behandlungsschema angewendet wurde. Am Ende der 24-wöchigen Studie standen die Tobramycin-Patienten demnach am Ende einer Off-Phase. Ziel der klinischen Prüfung war, die Nicht-Unterlegenheit von Colistimethat-Natrium Pulver gegenüber der Tobramycinlösung zu zeigen, definiert als maximal 3\% Unterschied zwischen den Behandlungsgruppen. Tatsächlich war der Verlauf der FEV1 unter Colistimethat-Natrium und unter Tobramycin-Lösung vergleichbar (Differenz Colistin-Tobramycin-0,97\% des FEV1-Solls). Dies galt sinngemäß auch für die forcierte Vitalkapazität FVC. In diesem Setting war Colistimethat-Natrium der Tobramycinlösung also nicht unterlegen.

Zum Tobramycin-Pulver, das zweimal täglich in einer Dosis von $112 \mathrm{mg}$ inhaliert wird, wurden unterschiedliche Vergleiche angestellt. Da Tobramycin als Inhalationslösung bereits seit Jahren auf dem Markt und seine Wirksamkeit bewiesen war, waren aus ethischen Gründen keine Langzeitstudien mit dem Pulver im Vergleich zu Placebo möglich. Dennoch wurde in zwei Studien kurzzeitig eine Placebo-Gruppe mitgeführt, und zwar über den ersten 28-tägigen Behandlungszyklus.

In der aktuellen EDIT-Studie stieg nach 4 Wochen TobramycinPulver die FEV1 in Prozent des Solls relativ um 5,9\% stärker gegenüber dem Ausgangswert an als unter Placebo. Die mittleren Veränderungen nach Tobramycin bzw. nach Placebo betrugen $+8,2$ bzw. $+2,3 \%$.

Im ersten Behandlungszyklus der EVOLVE-Studie wurde ebenfalls die Wirksamkeit von Tobramycin-Pulver mit der von Placebo verglichen [11]. Nach 28 Tagen Tobramycin-Pulver war die FEV1 in Prozent des Solls um 13,3\% stärker angestiegen als unter Placebo ( $\mathrm{p}=0,0016)$, wobei die Placebogruppe keine Änderung gegenüber dem Ausgangswert zeigte. Danach wechselten alle Studienteilnehmer in die offene Phase der Studie. Sie inhalierten in den folgenden 12 Wochen zweimal vier Wochen mit TobramycinPulver, mit einer dazwischen liegenden vierwöchigen Pause (On-Off-On). Auch nach Übergang von Placebo auf Tobramycin verbesserte sich die FEV1 signifikant.

In der EAGER-Studie wurde die Inhalation von Pulver über 24 Wochen mit $300 \mathrm{mg}$ Tobramycin-Lösung verglichen, um die Sicherheit der neuen Pulverformulierung zu evaluieren [25]. Beide Gruppen inhalierten also dasselbe Antibiotikum in denselben 28 Tage On-Off-Zyklen, nur Formulierung und Inhalationsgeräte (Podhaler ${ }^{\circledR}$ versus Kompressionsvernebler) waren unterschiedlich. Mehr als $80 \%$ der Teilnehmer hatten schon vor der Studie mit Tobramycin-Lösung inhaliert. Nach drei On-Off-Zyklen waren in Woche 24 keine signifikanten Unterschiede der FEV1 zwischen den Gruppen festzustellen; mit dem Podhaler ${ }^{\circledR}$ waren die relativen FEV1-Anstiege 1,1\% höher als nach Tobramycin-Lösung. Diese Studien zeigen, dass alle drei Arzneimittel in Pulverform die Lungenfunktion verbessern. Mannitol Pulver verbessert die FEV1 stärker als Placebo. Ein 28-Tage-Zyklus mit TobramycinPulver führt ebenfalls zu einem stärkeren Anstieg der FEV1 als Placebo. Tobramycin-Pulver über drei Behandlungszyklen führt zu einem vergleichbaren Verlauf der FEV1 wie unter Tobramy- 
Tab.4 Unerwünschte Ereignisse in den großen Phase-3-Studien zur Pulverinhalation.

\begin{tabular}{|c|c|c|c|c|c|c|c|c|c|c|}
\hline & \multicolumn{2}{|c|}{ Colistimethat-Natrium } & \multicolumn{2}{|c|}{ Tobramycin Pulver } & \multicolumn{2}{|c|}{ Tobramycin Pulver } & \multicolumn{2}{|c|}{ Tobramycin Pulver } & \multicolumn{2}{|l|}{ Mannitol } \\
\hline Acronym & FREEDC & & EAGER & & EVOLVE & & EDIT & & & \\
\hline Gruppe & $\mathrm{CDPI}^{*}$ & TIS* & TIP* & TIS & TIP & Placebo & TIP & Placebo & Mannitol & Kontrolle \\
\hline Formulierung & Pulver & Lösung & Pulver & Lösung & Pulver & Pulver & Pulver & Pulver & Pulver & Pulver \\
\hline n Pat & 186 & 193 & 308 & 209 & 46 & 49 & 30 & 32 & 361 & 239 \\
\hline \multicolumn{5}{|c|}{ Unerwünschte Ereignisse (\% der Patienten) } & \multicolumn{6}{|c|}{ | Zyklus 1 | } \\
\hline Husten & 16 & 10 & 48 & 31 & 13 & 27 & 10 & 0 & 21 & 17 \\
\hline $\begin{array}{l}\text { Verschlechterung/ } \\
\text { Exazerbation }\end{array}$ & & & 34 & 30 & 11 & 12 & 3 & 0 & 37 & 40 \\
\hline produktiver Husten & 5 & 6 & 18 & 20 & 2 & 8 & & & & \\
\hline Fieber & & & 16 & 12 & 7 & 4 & & & & \\
\hline Dyspnoe & 7 & 8 & 16 & 12 & & & & & & \\
\hline Dysphonie & & & 14 & 4 & 4 & 0 & 3 & 3 & & \\
\hline $\begin{array}{l}\text { Reizung/Schmerzen im } \\
\text { Rachen }\end{array}$ & 8 & 5 & 14 & 11 & 11 & 0 & & & 12 & 8 \\
\hline Hämoptysis & & & 13 & 12 & & & 3 & 0 & 13 & 13 \\
\hline Kopfschmerzen & & & 11 & 12 & 0 & 6 & & & 18 & 21 \\
\hline Bakterien im Sputum & & & & & & & & & 11 & 11 \\
\hline schlechter Geschmack & 11 & 5 & & & 7 & 2 & 3 & 0 & & \\
\hline Schwerhörigkeit & & & & & & & 10 & 6 & & \\
\hline Übelkeit & & & 8 & 10 & & & & & & \\
\hline $\begin{array}{l}\text { Infektion der unteren } \\
\text { Atemwege }\end{array}$ & 6 & 7 & & & & & 0 & 3 & 6 & 10 \\
\hline
\end{tabular}

* CDPI: Colistin Trockenpulver, TIP: Tobramycin Pulver, TIS: Tobramycin Lösung

cin-Inhalationslösung. Auch täglich über 24 Wochen inhaliertes Colistimethat-Natrium Pulver ist ähnlich wirksam, bezogen auf die FEV1 wie drei Zyklen Tobramycin-Lösung.

\section{Andere Wirkungen}

Die osmotische Wirkung von Mannitol bewirkt eine Hydrierung des epithelialen Flüssigkeitsfilms und dadurch eine Steigerung der mukoziliären Clearance [14]. Bei CF-Patienten veränderten sich nach Inhalation von Mannitol die Eigenschaften des Sputums. So nimmt der prozentuale Gehalt an Feststoffen ab und damit umgekehrt der Wassergehalt zu, und die Oberflächenspannung des Sputums verringert sich [26]. Diese Effekte traten bereits in der ersten Stunde nach Pulverapplikation auf und wurden bis 12 Stunden nach Inhalation dokumentiert. Mannitol kann auf diese Weise zu einer effektiveren Sekretclearance beitragen.

Die beiden Antibiotika in Pulverform, Colistimethat-Natrium und Tobramycin, supprimieren das Wachstum von $P$. aeruginosa im Sputum. Nach 28 Tagen Tobramycin nahm die Bakteriendichte mukoider Pseudomonaden um durchschnittlich 2,61 $\log _{10}$ kolonieformende Einheiten (colony forming units, CFU) pro Milliliter Sputum ab, nach Placebo waren es nur 0,43 $\log _{10}$ CFU/ml [11]. Auch nicht-mukoide Phänotypen nahmen signifikant stärker ab als unter Placebo. In der placebokontrollierten EDIT-Studie fiel die Bakteriendichte um $1,2 \log _{10} \mathrm{CFU} / \mathrm{ml}$ [27]. Die Publikation der Phase-3-Studie zu Colistin enthält keine Angaben zur Bakteriendichte [24]. Die Sputumkonzentrationen des Antibiotikums lagen jedoch etwa 20mal höher als die mittleren Hemmkonzentrationen $\mathrm{MIC}_{50}$ der Pseudomonaden.

\section{Verträglichkeit und Sicherheit}

Zur Verträglichkeit der Substanzen stellt $\bullet$ Tab. 3 die Häufigkeit unerwünschter Ereignisse aus den großen Phase-3-Studien dar. In Abhängigkeit von der Dauer der Studie wurden bei bis zu $94 \%$ der Patienten unerwünschte Ereignisse dokumentiert. Unter Mannitol und Colistin kam es bei 12\% bzw. 11\% der Studienteilnehmer zum vorzeitigen Abbruch der Studie, im Vergleich zu 3\% bzw. 6\% in den jeweiligen Vergleichsgruppen. Schwerwiegende unerwünschte Ereignisse (SUEs) wurden in der Mannitol-Studie über 26 Wochen bei $27 \%$ der Kontrollpatienten dokumentiert, wobei es sich bei $17 \%$ um Verschlechterungen des Zustands bzw. Exazerbationen handelte. Dagegen traten SUEs in den Antibiotika-Studien seltener auf.

Die häufigsten unerwünschten Ereignisse, die in denselben Studien auftraten, sind in Tab. 4 zusammengefasst. Es dominierten Husten (je nach Studie 10 bis $48 \%$ ) und respiratorische Exazerbationen. Schmerzen im Rachen, Heiserkeit, Hämoptysen und Kopfschmerzen traten bei gut 10\% der Studienteilnehmer auf.

\section{Nebenwirkungen}

Die Fachinformationen der drei Arzneimittel listen eine Vielzahl unterschiedlicher Nebenwirkungen auf. Die sehr häufigen $\mathrm{Ne}$ benwirkungen, die bei $\geq 10 \%$ der Anwender beobachtet wurden, sind ebenso wie die häufigen (bei 1-9,9\%) in $\bullet$ Tab. 5 zusammengestellt.

Die Mukoviszidose selbst führt zu Symptomen und Problemen, die in Arzneimittelstudien als unerwünschtes Ereignis dokumentiert werden müssen. Ein Beispiel ist Husten mit oder ohne Auswurf. Er wird in der Fachinformation als Nebenwirkung aufgeführt, wenn das Ereignis in Studien unter Verum deutlich häufiger auftrat als in der Placebo-Gruppe. Husten wird als sehr häufige Nebenwirkung bei allen drei Arzneimitteln genannt. Dies ist mit großer Wahrscheinlichkeit auf die Applikation als Pulver zurückzuführen. Unter den Antibiotika-Pulvern traten bei mehr als $10 \%$ der Anwender zusätzlich Dyspnoe, Heiserkeit und Rachenbeschwerden auf. Bei Colistin wurden außerdem Geschmacksstörungen registriert, bei Tobramycin Hämoptysen und Fieber. 


\begin{tabular}{|c|c|c|}
\hline $\begin{array}{l}\text { Colistimethat-Natrium } \\
\text { (Colobreathe }^{\circledR} \text { ) }\end{array}$ & $\begin{array}{l}\text { Tobramycin Pulver } \\
\text { (TOBI Podhaler }{ }^{\circledR} \text { ) }\end{array}$ & $\begin{array}{l}\text { Mannitol } \\
\text { (Bronchitol }^{\circledast} \text { ) }\end{array}$ \\
\hline \multicolumn{3}{|l|}{ sehr häufig ( $\geq 1: 10$ ) } \\
\hline Husten & Husten mit und ohne Auswurf & Husten \\
\hline Dyspnoe & Dyspnoe & \\
\hline Dysphonie & Dysphonie & \\
\hline Rachenreizung & Oropharyngealschmerzen & \\
\hline \multirow[t]{2}{*}{ Geschmacksstörung } & Fieber & \\
\hline & Hämoptyse & \\
\hline \multicolumn{3}{|l|}{ häufig ( $\geq 1: 100$ bis $<1: 10$ ) } \\
\hline Hämoptyse & & Hämoptyse \\
\hline Asthma & & Asthma \\
\hline Bronchospasmus & Bronchospasmus & \\
\hline Keuchen & Pfeifendes Atmen & Giemen \\
\hline produktiver Husten & & Husten mit Auswurf \\
\hline Lungenknistern & Rasselgeräusche & \\
\hline Erbrechen & Erbrechen & Erbrechen (auch nach dem Husten) \\
\hline Übelkeit & Übelkeit & \\
\hline \multirow[t]{3}{*}{ Tinnitus } & Tinnitus & \\
\hline & $\begin{array}{l}\text { Beschwerden im Brustkorb und } \\
\text { muskuloskeletale Brustschmerzen }\end{array}$ & Thoraxbeschwerden \\
\hline & Rachenreizung & $\begin{array}{l}\text { Rachenreizung, Rachen- und Kehl- } \\
\text { kopfschmerzen }\end{array}$ \\
\hline Gleichgewichtsstörung & Gehörverlust & Zustand verschlimmert \\
\hline Infektion der unteren Atemwege & Geschmacksstörung & Bakterien im Sputum identifiziert \\
\hline Arthralgie & Epistaxis & \\
\hline Pyrexie & Nasenverstopfung & \\
\hline Asthenie & Diarrhoe & \\
\hline Müdigkeit & Ausschlag & \\
\hline \multicolumn{3}{|l|}{$\begin{array}{l}\text { Verminderung des forcierten } \\
\text { exspiratorischen Volumens }\end{array}$} \\
\hline Kopfschmerz & & \\
\hline
\end{tabular}

Tab.5 In den Fachinformationen deklarierte Nebenwirkungen der drei Arzneimittel.
Unter den häufigen (1:10 bis 1:100) pulmonalen Nebenwirkungen sind Bronchospasmus, Asthma, erschwerte Atmung, Thoraxbeschwerden, Hämoptysen und produktiver Husten verzeichnet. Auch Erbrechen und Übelkeit zählen zu den häufigen Nebenwirkungen. Für die Antibiotika Tobramycin und Colistin sind Übelkeit und Tinnitus dokumentiert, für Colistin Gleichgewichtsstörungen und für Tobramycin-Pulver Gehörverlust und Geschmacksstörungen. Weitere Nebenwirkungen und ihre Verteilung auf die Arzneimittel können der Tab.5 entnommen werden.

\section{Anwendung der Pulverinhalatoren \\ $\nabla$}

Das optimale Inhalationsmanöver ist eine möglichst lange, tiefe und gleichmäßige Einatmung. Bei diesen Pulverinhalatoren ist es falsch, kräftig, schnell und kurz einzuatmen. Ein solches Atemmanöver provoziert außerdem einen Hustenreiz.

Nach der tiefen Einatmung soll der Patient 5-10 Sekunden den Atem anhalten (bzw. so lange, wie es ohne Anstrengung möglich ist). Erst danach erfolgt eine normale, ruhige Ausatmung. Auf diese Weise wird die pulmonale Deposition des Arzneimittels verbessert. Wichtig ist, den Inhalator beim Ausatmen seitlich vom Kopf zu halten, damit keine Feuchtigkeit aus der Ausatemluft in das Gerät gelangt. Bei Mannitol und Tobramycin muss dasselbe Manöver 10mal bzw. 4mal wiederholt werden.

In jedem Fall ist es wichtig dafür zu sorgen, dass Inhalator und Medikamentenpulver trocken bleiben. Die Geräte dürfen nicht mit Wasser gereinigt werden. Sollte Wasser ins Gerät eingedrun- gen sein, kommt bei Tobramycin das Podhaler ${ }^{\circledR}$ Ersatzgerät zum Einsatz, das in jeder Monatspackung enthalten ist.

Die unterschiedlichen inhalativen Medikamente richtig anzuwenden, ist für Patient und Behandler eine Herausforderung. Bei chronischen Atemwegserkrankungen inhalieren die Patienten häufig mehrere verschiedene Medikamente. Für jedes Device und für jede Substanz müssen sie die speziellen Atemmanöver erlernen und sicher anwenden können.

Anders als bei oralen Medikamenten reicht es nicht, wenn der Arzt das Arzneimittel einfach nur verordnet. Der Patient muss auch geschult werden. Wichtige Unterlagen hierfür sind die Gebrauchsinformationen der einzelnen Präparate. In vielen Ambulanzen werden zusätzlich Schulungsmaterialien der pharmazeutischen Hersteller verwendet. Die Deutsche Atemwegsliga hat eine Serie von Videos im Internet bereitgestellt, in denen für jedes Device die richtige Inhalation gezeigt wird [28]. Auch die neuen Pulverpräparate für Mukoviszidose wurden inzwischen in diese Serie aufgenommen.

Grundsätzlich sollte die erste Dosis unter Aufsicht verabreicht werden, ggf. nach Inhalation eines Bronchodilatators; bei Mannitol ist eine vorherige Initialdosis-Testung nötig.

Eine zentrale Rolle beim Training der Patienten kommt den Physiotherapeuten zu. Meist sind sie es, die mit dem Patienten das richtige Inhalieren trainieren. Dies ist nicht nur dann erforderlich, wenn ein neues Medikament verordnet wird, sondern es ist auch sinnvoll, in regelmäßigen Abständen zu überprüfen, wie gut der Patient die unterschiedlichen Inhalationsmanöver beherrscht. 


\section{Fazit}

Zur Pulverinhalation bei Mukoviszidose wurden mit neuen Technologien lungengängige Partikel entwickelt, mit denen auch größere Arzneimittelmengen pulmonal deponiert werden können. Im Unterschied zu konventionellen Pulverinhalatoren liegen die Massen der Einzeldosen bei den neuen Arzneimitteln 10- bis 100fach höher als bei Bronchodilatatoren oder inhalativen Steroiden.

Die Pulverinhalation von Antibiotika und von Mannitol hat sich in Studien als wirksam und verträglich erwiesen. Im Vergleich zur Feuchtinhalation über Kompressions- oder Membranvernebler ergeben sich für den Patienten vor allem praktische Vorteile. Die häufigste Nebenwirkung bei mehr als 10\% der Patienten ist Husten. Bei Mannitol ist vor Therapiebeginn eine vorherige Initialdosis-Testung nötig.

Wie bei allen inhalativen Anwendungen von Medikamenten ist das sorgfältige Training der Patienten unerlässlich. Hier liegt eine wichtige Aufgabe für die Physiotherapeuten, die bei Mukoviszidosepatienten die inhalative Therapie ohnehin regelmäßig überwachen sollten.

\section{Interessenkonflikt}

\section{$\nabla$}

Für das Verfassen dieses Manuskripts erhielt die Autorin ein Honorar von der Novartis Pharma GmbH.

\section{Literatur}

1 Griese M, Glöckner-Pagel J. Aerosoltherapie und Applikation. In: Griese M, Nicolai T (Hrsg). Praktische Pneumologie in der Pädiatrie - Therapie. Stuttgart: Georg Thieme Verlag KG; 2013: 53-61

2 Tiddens HA, Geller DE, Challoner P et al. Effect of dry powder inhaler resistance on the inspiratory flow rates and volumes of cystic fibrosis patients of six years and older. J Aerosol Med 2006; 19: 456-465

3 Voshaar T, App EM, Berdel D et al. Empfehlungen für die Auswahl von Inhalationssystemen zur Medikamentenverabreichung [Recommendations for the choice of inhalatory systems for drug prescription]. Pneumologie 2001; 55: 579-586

4 Ryan G, Singh M, Dwan K. Inhaled antibiotics for long-term therapy in cystic fibrosis. Cochrane Database Syst Rev 2011; 3: CD001021

5 Govoni M, Poli G, Acerbi D et al. Pharmacokinetic and tolerability profiles of tobramycin nebuliser solution $300 \mathrm{mg} / 4 \mathrm{ml}$ administered by PARI eFlow $((\mathrm{R}))$ rapid and PARI LC Plus((R)) nebulisers in cystic fibrosis patients. Pulm Pharmacol Ther 2013; 26: 249-255

6 Geller DE, Weers J, Heuerding S. Development of an Inhaled Dry-Powder Formulation of Tobramycin Using PulmoSphere Technology. J Aerosol Med Pulm Drug Deliv 2011; 24: 175-182

7 Weers J, Clark A, Challoner P. High dose inhaled powder delivery: challenges and techniques. In: Dalby RN, Byron PR, Peart J et al. (Hrsg) Respiratory Drug Delivery IX. Palm Desert, CA, USA: Davis Healthcare International Publishing; 2004: $281-288$

8 Newhouse MT, Hirst PH, Duddu SP et al. Inhalation of a dry powder tobramycin PulmoSphere formulation in healthy volunteers. Chest 2003 ; 124: $360-366$

9 Novartis Europharm Limited. Zusammenfassung der Merkmale des Arzneimittels (EPAR TOBI Podhaler Product Information). 20. 07. 2011
10 Geller DE, Konstan MW, Smith J et al. Novel tobramycin inhalation powder in cystic fibrosis subjects: Pharmacokinetics and safety. Pediatr Pulmonol 2007; 42: 307-313

11 Konstan MW, Geller DE, Minic P et al. Tobramycin inhalation powder for $P$. aeruginosa infection in cystic fibrosis: The EVOLVE trial. Pediatr Pulmonol 2010; 46: 230-239

12 Glover W, Chan HK, Eberl S et al. Lung deposition of mannitol powder aerosol in healthy subjects. J Aerosol Med 2006; 19: $522-532$

13 Anderson SD, Brannan J, Spring J et al. A new method for bronchial-provocation testing in asthmatic subjects using a dry powder of mannitol. Am J Respir Crit Care Med 1997; 156: 758 - 765

14 Daviskas E, Anderson SD, Brannan JD et al. Inhalation of dry-powder mannitol increases mucociliary clearance. Eur Respir J 1997; 10: $2449-2454$

15 Robinson M, Daviskas E, Eberl S et al. The effect of inhaled mannitol on bronchial mucus clearance in cystic fibrosis patients: a pilot study. Eur Respir J 1999; 14: 678-685

16 Pharmaxis Pharmaceuticals Limited. Zusammenfassung der Merkmale des Arzneimittels (EPAR Bronchitol Product Information). 13. 04. 2012

17 Stass $H$, Nagelschmitz J, Willmann $S$ et al. Inhalation of a dry powder ciprofloxacin formulation in healthy subjects: a phase I study. Clin Drug Investig 2013; 33: 419-427

18 Claus SC. Novel Dry Powder Inhalation System Based on Dispersion of Lyophilisates. Fakultät für Chemie und Pharmazie, Ludwig-Maximilians-Universität München; 2012

19 Magni P. Single-dose inhalers for capsules: A consolidated tradition with ample growth prospects. ONdrugDelivery 2012: 26-27

20 Glover W, Chan HK, Eberl $S$ et al. Effect of particle size of dry powder mannitol on the lung deposition in healthy volunteers. Int J Pharm 2008; 349: $314-322$

21 Montgomery AB, Abuan T, Yeager MA. Regulatory Aspects of Phase 3 Endpoints for New Inhaled Antibiotics for Cystic Fibrosis Patients with Chronic Pseudomonas aeruginosa Infections. J Aerosol Med Pulm Drug Deliv 2012; 25: 198 - 203

22 Konstan MW, Morgan WJ, Butler SM et al. Risk factors for rate of decline in forced expiratory volume in one second in children and adolescents with cystic fibrosis. J Pediatr 2007; 151: $134-139$

23 Bilton D, Bellon G, Charlton B et al. Pooled analysis of two large randomised phase III inhaled mannitol studies in cystic fibrosis. J Cyst Fibros 2013; 12 : $367-376$

24 Schuster A, Haliburn C, Doring G et al. Safety, efficacy and convenience of colistimethate sodium dry powder for inhalation (Colobreathe DPI) in patients with cystic fibrosis: a randomised study. Thorax 2013; 68: $344-350$

25 Konstan MW, Flume PA, Kappler M et al. Safety, efficacy and convenience of tobramycin inhalation powder in cystic fibrosis patients: The EAGER trial. J Cyst Fibros 2011; 10: 54-61

26 Daviskas E, Rubin BK. Effect of inhaled dry powder mannitol on mucus and its clearance. Expert Rev Respir Med 2013; 7: 65 - 75

27 Galeva I, Konstan W, Higgins $M$ et al. Tobramycin inhalation powder manufactured by improved process in cystic fibrosis: the randomized EDIT trial. Curr Med Res Opin 13 A.D; 29: 947-956

28 Knipel V, Criee CP, Windisch W. Korrekte Inhalationstherapie: Einweisung mittels Internet-verfügbarer Filmsequenzen. Eine Initiative der Deutschen Atemwegsliga e.V. [Correct inhalation therapy: instructions provided by Internet-based video screens. An initiative of the German Airway League]. Pneumologie 2013; 67: 157-161

29 Goldman M, Riethmueller J, Turay P. A 7-day open-label PK study to investigate the systemic absorption of a dry powder formulation of inhaled colistimethate sodium (CDPI) [Colobreathe ${ }^{\circledR} 1,662,500 \mathrm{IU}$ ] in adult, adolescent and paediatric cystic fibrosis subjects with chronic Pseudomonas aeruginosa lung infection [WS 5.5]. Journal of cystic fibrosis: official journal of the European Cystic Fibrosis Society 2013; 11 (Suppl. 01): 12

30 Pharmaxis Ltd.Australia. Australian Public Assessment Report for Mannitol. 1-3-2011. Australian Government, Department of Health and Ageing, Therapeutic Goods Administration 